\title{
Further Brain Magnetic Resonance Imaging (MRI) Delineation of Aicardi-Goutières Syndrome (AGS)
}

\author{
Amal Y. Kentab* \\ Department of Pediatric, Neurology Division, King Khalid University Hospital, King Saud University Riyadh, Saudi Arabia
}

Submission: January 19, 2019; Published: February 27, 2019

*Corresponding author: Amal Y. Kentab, Department of Pediatrics, Neurology division, King Khalid University Hospital, College of Medicine, King Saud University, Riyadh, Saudi Arabia

\begin{abstract}
Aicardi-Goutières syndrome (AGS) is a genetically heterogeneous disorder reported previously as an inherited congenital infection-like syndrome. It is characterized by an early onset progressive encephalopathy, brain calcification, leukodystophy, CSF chronic lymphocytosis, elevated CSF alpha-interferon, and negative results for common prenatal infections. It is autosomal-recessive or rarely autosomal-dominant and caused by mutations in one of several genes TREX1, RNASEH2A, RNASEH2B, and RNASEH2C, SAMHD1, IFIH1, and ADAR. The Magnetic resonance imaging findings of two male siblings diagnosed genetically with AGS 5 due to a homozygous mutation in SAMHD1 with very similar characteristics comprising profound psychomotor retardation, progressive microcephaly, spasticity, dystonic posturing, and tonic seizures are reported. The author reviewed the literature to delineate a specific MRI pattern of AGS.

Keywords: Aicardi-Goutières Syndrome; Neuro-radiologic findings; Magnetic Resonance Imaging (MRI); Leukodystrophy; SAMHD1 mutation

Abbreviations: AGS: Aicardi-Goutières Syndrome; MRI: Magnetic Resonance Imaging; CILS: Chronic Infection-Like Syndrome; MRI: Magnetic Resonance Imaging; VEP: Visual Evoked Potential; BAEP: Brain Auditory Evoked Potentials; CSF: Cerebrospinal Fluid; MRA: Magnetic Resonance Angiography
\end{abstract}

\section{Introduction}

Aicardi-Goutières (AGS) Syndrome is a rare genetically heterogeneous disorder. It was first described as a distinct Chronic Infection-Like Syndrome (CILS) with chronic CSF leukocytosis, basal ganglia calcification and a negative test for infection by Aicardi and Goutierés [1], Lanzi, et al. [2], and La Piana, et al. [3]. It was further distinguished from other CILS by the classical bilateral calcification of the basal ganglia, leukoencephalopathy and/or cerebral atrophy.

It has a prevalence of 1-5/10,000. Currently, seven different types of AGS are described as mutations in 7 genes have been identified TREX1, RNASEH2A, RNASEH2B, RNASEH2C, SAMHD1, ADAR, and IFIH1 [4]. AGS is generally inherited as an autosomal recessive trait although the dominant heterozygous gain of function mutations in TREX1, ADAR1, and all IFIH1 genes were observed [5]. Mutations in these genes enhance the production of type 1interferon and upregulation of interferon-stimulated genes. Consequently, disturbance of both cellular and immunological function occurs, which results in elevation of interferon- $\alpha$ both in the CSF and blood [5].

Typically affected patients show severe, subacute encephalopathy characterized by irritability, feeding problems, psychomotor regression or delay associated with epilepsy (53\% of cases), chilblain skin lesions on the extremities ( $43 \%$ of cases) and episodes of aseptic febrile illness (40\% of cases), progressive microcephaly, spasticity, and dystonia, often followed by death in early childhood $[2,6,5]$. It is phenotypically heterogeneous as it shows inter- and intrafamilial variations with a range of onsets between the first month of life usually with life-threatening illness and a less severe form described during late infancy with preservation of language skills and cognitive function, and a normal head circumference. The disease is rapidly fatal or progresses to a vegetative state [7].

Elevated IFN-alpha levels and CSF lymphocytosis are very frequent but not constant findings $(90 \%$ and $75 \%$ of cases, respectively) at the initial stage of the disease but tend to normalize or resolve within a few years [7]. Typical MRI features include early temporal lobe swelling followed by atrophy, early global cerebral atrophy and visible calcification [8].

Magnetic Resonance Imaging (MRI) findings of two male siblings diagnosed genetically with AGS 5 with very similar characteristics comprising profound psychomotor retardation, progressive microcephaly, spasticity, dystonic posturing, and tonic seizures are reported. The author reviewed the literature to delineate a specific MRI pattern of AGS. 


\section{Open Access Journal of Neurology \& Neurosurgery}

\section{Case Report}

\section{Patient 1}

This boy, currently 5 years old, was initially evaluated because of global developmental delay and seizures at the age of 5 months. He was the first child of healthy consanguineous Saudi parents. There was no family history of epilepsy or neurological disorders or neonatal deaths. He was a product of term pregnancy and delivered by emergency caesarean section due to meconium liquor. Head circumference at birth was 35.5 $\mathrm{cm}$ (at 50th percentile). He had mild, transient jaundice at birth. Since the early neonatal period, he was noticed to have extreme irritability and poor feeding. He had poor visual tracking and hearing with no social smile. At 4 months of age, he had poor head control, was not rolling and showed intermittent stiffness of his limbs. He developed tonic seizures and episodes of spontaneous clonic movements and staring at the age of 4 months. Epilepsy was resistant to multiple antiepileptic medications, but he was maintained on phenobarbitone, carbamazepine, topiramate, and clonazepam with reasonable control. He had profound developmental delay and inability to communicate or ambulate. Examination at the age of 5 months showed a head circumference of $32 \mathrm{~cm}$ (25th percentile), no response to visual stimuli, head lag, axial hypotonia, and extremity hypertonia with hyperreflexia in both arms and legs. Follow-up exam at one year of age showed progressive spastic quadriplegia, and he developed joint contractures despite oral baclofen and physiotherapy. Initial evaluation included a normal abdominal ultrasound and dilated ophthalmological examination. Brain MRI (at 4 months of age) showed multiple subcortical cysts, extensive bitemporal cystic lesions, and significant thinning of the brain stem and cerebellar atrophy.
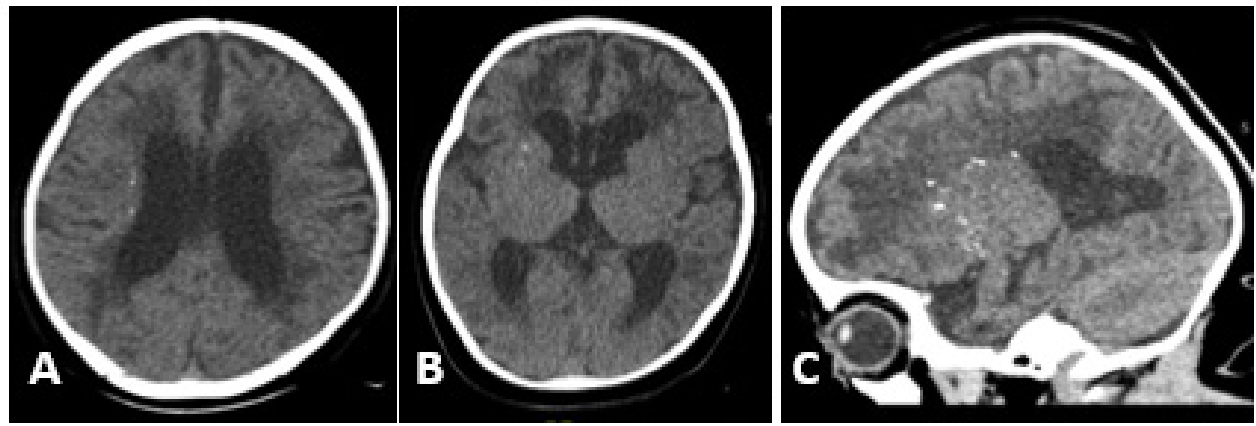

Figure 1: Brain CT scan ( Axial) at 4 months of age showing intracranial calcification (A) AT White matter and periventricular region ,( B) At Basal ganglia , ( Sagittal) (C ) With a periventricular distribution.
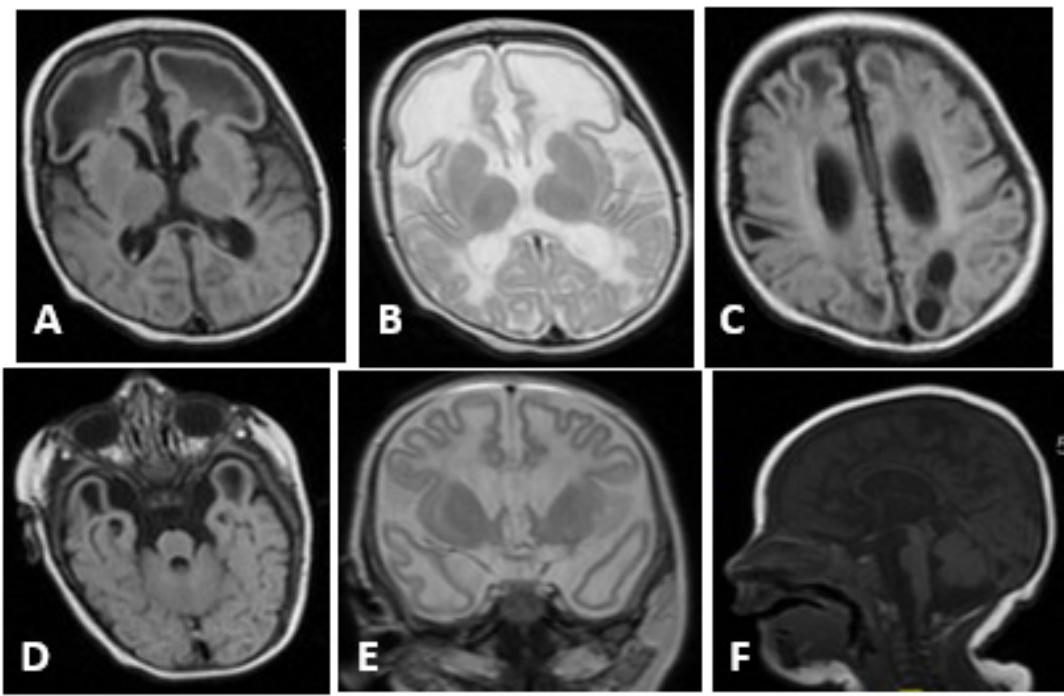

Figure 2: Brain MRI scan at 4 months of age with a typical pattern of AGS (A) Axial FLAIR imaging with hypo intensity of white matter (B) Axial T2-weighted image with hyperintensity of white matter (C) Axial FLAIR imaging with multiple subcortical cysts (D) Axial FLAIR imaging, and $(E)$ Coronal T2-weighted imaging with extensive bitemporal cystic lesions (F) Sagittal T1-weighted imaging with significant thinning of the brain stem and cerebellar atrophy

Brain CT scan during infancy showed calcification at Metabolic testing was normal and included the following: basal ganglia, and periventricular white matter (Figure 1, 2). serum amino acids, plasma very long-chain fatty acids, plasma 


\section{Open Access Journal of Neurology \& Neurosurgery}

acylcarnitine profile, serum biotinidase level, serum ammonia, lactate, and urine organic acids. Chromosomal microarray was normal. Electroencephalogram (EEG) revealed slow background and multifocal epileptiform discharges. Visual Evoked Potential (VEP) and Brain Auditory Evoked Potentials (BAEP) were normal. Cerebrospinal Fluid (CSF) testing at 4 months of age showed normal cell count, protein, glucose, lactate and amino acid content. A follow-up brain MRI at 4 years of age showed severely atrophic brain parenchyma with enlargement of ventricles, reduced bulk of cerebral white matter, thinning of corpus callosum and brainstem. Cystic changes were noted at bilateral frontal lobes, left parietal lobe, and tiny cysts in anterior temporal lobes (Figure 3). Brain Magnetic Resonance Angiography (MRA) obtained concurrently was normal. Further molecular analysis confirmed Aicardi-Goutieres syndrome 5 due to a homozygous mutation in SAMHD1 gene.
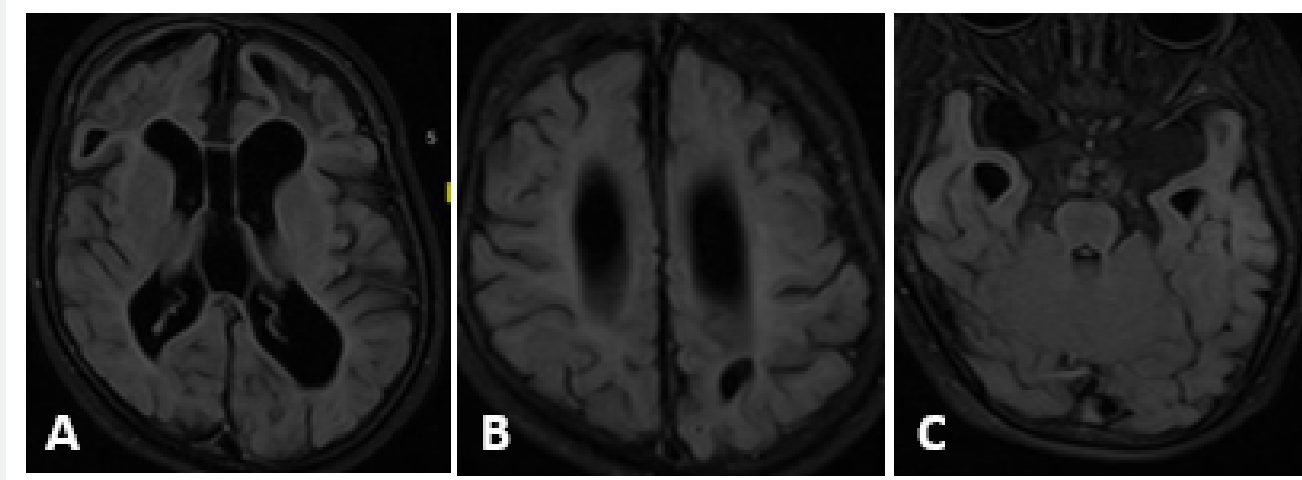

Figure 3: Brain MRI scan at 54 months of age with a typical pattern of AGS (A), (B) Axial FLAIR imaging with hypo intensity of white matter reduced white matter bulk, multiple subcortical cysts and cortical and subcortical atrophy with ventriculomegaly. (C) Coronal T2-weighted FLAIR imaging with extensive bitemporal cystic lesions.

\section{Patient 2}

Patient 2 was the younger brother of patient 1 . He was born at term by normal vaginal delivery following an uneventful pregnancy. Birth weight and head circumference were $3 \mathrm{~kg}$ (10th percentile) and $32 \mathrm{~kg}$ (at $5^{\text {th }}$ percentile), respectively. He presented with a similar picture like his brother, though the severity of symptoms was less. He had episodes of sterile pyrexia. At the age of 5 months, he experienced partial tonic seizures and EEG showed slow background activity with infrequent sharp- and slow- wave activity at the tempro-occipital region. Seizures were controlled by carbamazepine and topiramate. Cranial CT performed at 8 months showed brain atrophy with periventricular and basal ganglia calcification. On physical exam at $11 / 2$ years, he was noted to have a head circumference of 44 $\mathrm{cm}\left(<5^{\text {th }}\right.$ percentile $)$ with weight and length $<10$ th percentile. He had spastic quadriplegia with intermittent dystonia with no acquisition of motor skills.

EEG records showed background slowing associated with infrequent sharp waves in the tempro-occipital region. Abdominal ultrasound and ophthalmological examination showed normal results. Brain MRI showed atrophic changes in the cortex, cerebellum and brainstem, mild ventromegaly and white matter loss.

\section{Discussion}

Aicardi-Goutières syndrome is a rare type of inherited leukoencephalopathy with variable clinical manifestations. It has autoimmune manifestations and large vessel vasculitis. Molecular detection of specific gene mutations is required to confirm the diagnosis, but certain neuroradiologic patterns can be detected initially, which may facilitate more precise and earlier diagnosis of this rare but probably underdiagnosed syndrome [8].

Both siblings in this report had the classic clinical picture of neonatal onset AGS, with subacute encephalopathy manifested with irritability, feeding problems, psychomotor delay, and epilepsy. Episodes of sterile pyrexia were seen in the younger sib. No autoimmune manifestations were recorded (chilblain skin lesions, hepatitis, arthropathy, or cardiomyopathy). The older sibling had primary hypothyroidism. They end with the typical phenotype of progressive microcephaly, irritability, spastic quadriplegia, dystonia, and GT insertion for feeding. The younger sib had less severe symptoms with a much slower course consistent with the previously reported data of inter- and intrafamilial variation $[4,9]$.

A homozygous mutation in SAMHD1 gene at 20q11.23 confirmed the diagnosis of AGS 5. Currently prenatal diagnosis is feasible through molecular analysis of amniotic fluid or trophoblasts. Treatment is symptomatic, mainly for feeding problems, spasticity, dystonia, epilepsy, and irritability with sleep disturbance.

AGS is classified under neurological disorders with mainly leukodystrophy pattern on brain MRI [4]. Earlier studies have shown that the cardinal features for diagnosis are intracranial calcification (involving the basal ganglia and white matter), cystic leukodystrophy (predominantly frontotemporal) and early cortical-subcortical atrophy. It is often associated with atrophy of the corpus callosum, brain stem and cerebellum $[9,10]$. 


\section{Open Access Journal of Neurology \& Neurosurgery}

Brain calcifications were typically small, multifocal, and seen mainly in the basal ganglia (putamina), lobar white matter, and dentate nuclei, but the globi pallidi, thalami, deep and subcortical white matter may be involved as well $[8,11]$. It identifies correctly up to $94.44 \%$ of AGS with a sensitivity of $90.9 \%$ and a specificity of $96.9 \%$.

The subcortical and deep white matter typically are abnormal and appear as hypodense lesions on CT, hypo intense on T1-weighted image and high signal intensity on T2-weighted image or FLAR image. These abnormalities are either diffuse or with anteriorposterior gradient mainly lobar with early involvement of subcortical arcuate fibers and with relative sparing of the corpus callosum, the capsules, and the optic radiations [12]. Imaging studies may also show ventriculomegaly, usually progressive, secondary to progressively reduced volume of white matter in the cerebral hemispheres [12]. In later stages of the disease, white matter may have signal intensity similar to CSF, particularly in the frontal lobes [12].

Both the calcification and white matter abnormalities increased in the first 2 years after diagnosis before becoming stable. Usually a significant degree of cerebral atrophy exists which remains substantially stable with time or worsens in some cases.

The cerebellum and brainstem may be small $[13,14]$, but the cerebellar white matter is often spared. Abdel-Salm, et al. [15] reported a rare finding of unilateral cerebellar hypoplasia in a patient with homozygous missense mutation in RNASEH2B gene.

The presence of deep white matter cysts in the temporal or frontal lobes has been reported only in a few patients in certain case series reports $[3,12,16]$. They represent a severe cystic degeneration of the white matter and were commonly observed in patients with TREX1 mutations [3]. Early on, patients may present with temporal lobe swelling followed by atrophy with temporal horn dilatation [8]. Furthermore, Olivieri, et al. [17] and Abdel-Salam GM, et al. [15] reported the presence of small and large porencephalic cysts, respectively.

Table 1: Neuroimaging findings: Aicardi-Goutieres syndrome.

\begin{tabular}{|c|c|}
\hline $\begin{array}{c}\text { Brain CT } \\
\text { scan }\end{array}$ & $\begin{array}{c}\text { Intracranial calcification - Basal gangalia, dentate } \\
\text { nuclei of the cerebellum, and periventricular white } \\
\text { matter }\end{array}$ \\
\hline \multirow{3}{*}{$\begin{array}{c}\text { Brain MRI } \\
\text { scan }\end{array}$} & $\begin{array}{c}\text { White matter abnormalities (Leukodystrophy) with } \\
\text { anterior-posterior gradients with hypo intensity on } \\
\text { T1-weighted image, and hyperintensity on T2-weighted } \\
\text { and FLAIR images. Sparing cerebellar white matter }\end{array}$ \\
\cline { 2 - 3 } & $\begin{array}{c}\text { Extensive bifrontal or bitemporal cystic lesions } \\
\text { Diffuse cerebral and subcortical atrophy, deep white } \\
\text { matter loss, progressive ventricular dilatation }\end{array}$ \\
\cline { 2 - 3 } & $\begin{array}{c}\text { Significant thinning of the brain stem and cerebellar } \\
\text { atrophy. }\end{array}$ \\
\hline $\begin{array}{c}\text { Brain MRA } \\
\text { scan }\end{array}$ & $\begin{array}{c}\text { Large vessels vasculitis, developmental vascular } \\
\text { variants (noted with SAMHD 1 mutation) }\end{array}$ \\
\hline
\end{tabular}

A recent study by Al Mutiri F, et al. [18] described the neuroimaging findings in a series of 24 patients; leukoencephalopathy in $83 \%$, cerebral atrophy in $75 \%$, and calcification in $54 \%$. Thinning of corpus callosum as well as ventromegaly in the absence of hydrocephalus was also seen but to a lesser extent. Only one patient was reported with white matter cysts. Follow-up MRI showed improvement in myelination and a decreased degree of atrophy with increasing duration of AGS.

In AGS 5 with SAMHD1 mutation, it is expected to have some large vessels vasculitis with some vascular developmental variants $[4,5]$, but brain MRA was normal for both siblings. Follow-up of our patients showed further cerebral and white matter atrophy with the collapse of previously reported cysts. The typical pattern of MRI found in AGS (Table 1) helps to differentiate it from other causes of early infantile leukoencephalopathy (Table 2).

Table 2: Differential diagnosis of AGS - early infantile leukodystrophies with and without temporal lobe cysts.

\begin{tabular}{|c|}
\hline Congenital infection such as cytomegalovirus (CMV) or rubella \\
Alexander disease (AxD) \\
\hline Mitochondrial leukoencephalopathies \\
\hline Megalencephalic leukodystrophy with cysts (MLC) \\
\hline $\begin{array}{c}\text { Antenatal onset Vanishing white matter with e IF2B mutations } \\
\text { (VWM) }\end{array}$ \\
\hline $\begin{array}{c}\text { CoatsPlus disease / leukoencephalopathy with Calcification and cyst } \\
\text { (CP/LCC) }\end{array}$ \\
\hline Cystic leukoencephalopathy without megalencephaly due to \\
RNASET2 mutations \\
\hline Various types of congenital muscular dystrophy (FCMD, Walker \\
Warburg syndrome, MDCID)
\end{tabular}

An algorithm of such differential diagnosis with and without temporal lobe swelling was reported by Vanderver A, et al. [8] to differentiate it from AGS based on MRI analysis. With increased availability and high specificity of the MRI, detection of calcification on CT scan is no longer essential for the diagnosis of AGS.

In conclusion, AGS is an underdiagnosed hereditary leukodystrophy syndrome with a typical clinical picture of early irritability, psychomotor retardation, and microcephaly as well as combined pyramidal and extrapyramidal manifestations. This report highlights the importance of early recognition of the typical MRI pattern of AGS that can help in clinical diagnosis before confirmation by molecular genetic studies, especially in patients with microcephaly and intracranial calcification.

\section{Acknowledgement}

This work was supported by the College of Medicine, Research Center, Deanship of Scientific Research, King Saud University.

\section{Conflict of Interest}

The author declares no conflict of interest. 


\section{References}

1. Aicardi J, Goutieres F (1984) A progressive familial encephalopathy in infancy with calcifications of the basal ganglia and chronic cerebrospinal fluid lymphocytosis. Ann Neurol 15(1): 49-54.

2. Lanzi G, Fazzi E, D Arrigo S (2002) Aicardi-Goutieres syndrome: a description of 21new cases and a comparison with the literature. Eur J Paediatr Neurol 6(supplA): A9-A22.

3. La Piana R, Uggetti C, Roncarolo F, et al. (2016) Neuroradiologic patterns and novel imaging findings in Aicardi-Goutieres syndrome. Neurology 86(1): 28-35.

4. Crow YJ, Chase DS, Lowenstein Schmidt J, Forte GM, Gornall HL, et al. (2015) Characterization of human disease phenotypes associated with mutations in TREX1, RNASEH2A, RNASEH2B, RNASEH2C, SAMHD1, ADAR, and IFIH1. Am J Med Genet A 167A (2): 296-312.

5. Livingston JH, Crow YJ (2016) Neurologic phenotypes associated with mutations in TREX1, RNASEH2A, RNASEH2B, RNASEH2C, SAMHD1, ADAR1, and IFIH1: Aicardi-Goutières syndrome and beyond. Neuropediatrics 47(6): 355-360.

6. Abdel-Salam GMH, El-Kamah GY, Rice GI, et al. (2010) Chilblains as a diagnostic sign of Aicardi-Goutières syndrome. Neuropediatrics 41(1):18-23.

7. Stephenson JB (2008) Aicardi-Goutieres syndrome (AGS). Eur J Paediatr Neurol 12(5): 355-358.

8. Vanderver A, Prust M, Kadom N, Demarest S, Crow YJ, et al. (2014) Early-onset Aicardi-Goutieres syndrome: magnetic resonance imaging (MRI) pattern recognition. J Child Neurol 30(10): 1343-1348.

9. Rice G, Patrick T, Parmar R, Taylor CF, Aeby A, et al. (2007) Clinical and molecular phenotype of Aicardi-Goutières syndrome. Am J Hum Genet 81(14): 713-725.
10. Fazzi E, Cattalini M, Orcesi S, Tincani A, Andreoli L, et al. (2013) Aicardi-Goutieres syndrome, a rare neurological disease in children: a new autoimmune disorder? Autoimmun Rev 12: 506-509.

11. Goutières F (2005) Aicardi-Goutières syndrome. Brain Dev 27: 201206.

12. Uggetti C, La Piana R, Orcesi S, Egitto MG, Crow YJ, et al. (2009) Aicardi-Goutieres syndrome: neuroradiologic findings and follow-up. AJNR Am J Neuroradiol 30(10): 1971-1976.

13. Vivarelli R, Grosso S, Cioni M, Galluzzi P, Monti L, et al. (2001) Pseudo-TORCH syndrome or Baraitser-Reardon syndrome: diagnostic criteria. Brain Dev 23(1): 18-23.

14. Reardon W, Hockey A, Silberstein P, Kendall B, Farag TI, et al. (1994) Autosomal recessive congenital intrauterine infection-like syndrome of microcephaly, intracranial calcification, and CNS disease. Am J Med Genet 52(1): 58-65.

15. Abdel-Salam GM, Abdel-Hamid MS, Mohammad SA, Abdel-Ghafar SF, Soliman DR, et al. (2017) Aicardi-Goutieres syndrome: unusual neuro-radiological manifestations. Metab Brain Dis 32(3): 679-683.

16. Rossler L, Ludwig-Seibold C, Thiels C, Schaper J (2012) Aicardi-Goutières syndrome with emphasis on sonographic features in infancy. Pediatr Radiol 42(8): 932-940.

17. Olivieri I, Cattalini M, Tonduti D, La Piana R, Uggetti C, et al. (2013) Dysregulation of the immune system in Aicardi-Goutières syndrome: another example in a TREX1-mutated patient. Lupus 22(10): 10641069.

18. Al Mutairi F, Alfadhel M, Nashabat M, El-Hattab AW, Ben-Omran T, et al. (2018) Phenotypic and Molecular Spectrum of Aicardi-Goutières Syndrome: A Study of 24 Patients. Pediatr Neurol 78: 35-40.

\section{Your next submission with Juniper Publishers} will reach you the below assets

- Quality Editorial service

- Swift Peer Review

- Reprints availability

- E-prints Service

- Manuscript Podcast for convenient understanding

- Global attainment for your research

- Manuscript accessibility in different formats

( Pdf, E-pub, Full Text, Audio)

- Unceasing customer service

Track the below URL for one-step submission

https://juniperpublishers.com/online-submission.php 